\title{
Modal Appropriation for Use with In-Operation Modal Analysis
}

\author{
M. Abdelghani' ${ }^{1}$ and D. J. Inman ${ }^{2}$ \\ ${ }^{1}$ ISSAT Sousse, Avenue Ibn Khaldoun, Taffala, 4003 Sousse, Tunisia \\ ${ }^{2}$ University of Michigan, Ann Arbor, MI 48109, USA \\ Correspondence should be addressed to M. Abdelghani; mahera@lycos.com \\ Received 30 May 2014; Revised 21 November 2014; Accepted 25 November 2014 \\ Academic Editor: Chao Tao
}

Copyright (C) 2015 M. Abdelghani and D. J. Inman. This is an open access article distributed under the Creative Commons Attribution License, which permits unrestricted use, distribution, and reproduction in any medium, provided the original work is properly cited.

\begin{abstract}
We propose in this paper a numerical modal appropriation method for use with in-operation modal analysis (INOPMA). The key idea is to realize that the correlation sequence of the system output is the sum of decaying sinusoids with a certain phase shift and therefore it may be considered as an impulse response. The method is based on performing a numerical convolution of a single sine wave force with the system output correlation sequence. The steps are then similar to the classical modal appropriation method, although the characteristic frequencies are different. This approach is validated and compared to a subspace method on simulated data as well as on experimental data and it is shown that INOPMA outperforms the subspace method.
\end{abstract}

\section{Introduction}

Classical modal parameter identification methods are usually based on frequency response functions or impulse response functions that require measurements of the input force and the resulting response. However, in some practical situations, modal parameters must be extracted from response measurements only. For example, for large structures (such as bridges, offshore platforms, and wind turbines), it is very difficult and sometimes impossible to measure actual excitation (such as wind, road noise, and wave excitation). The large amount of energy necessary to induce structural vibrations may cause local damage and excitation becomes very difficult to generate. Moreover, the actual operating conditions may differ significantly from the laboratory conditions. Therefore, in these applications, the system identification approach must be done on the basis of in-operation output-only data. The method of in-operation modal analysis has gained considerable attention in recent years. There have been several different approaches to estimate modal parameters from outputonly data. They include peak-picking from power spectral density functions [1], least squares curve fitting technique [2], subspace methods $[3,4]$, and the natural excitation technique (NExT) [5] using cross correlation functions instead of impulse response functions.
On the other hand, one of the most powerful methods for modal identification is the modal (or force) appropriation method which uses one sine signal to generate forces at different points of the structure and adjusts the relative values of those forces so as to isolate a single mode. Such tests provide very accurate information on the mode shapes, which is then complemented by specific tests to determine the modal damping [6].

Inspired by this last method, we propose in this paper a modal appropriation based method for use with in-operation modal analysis (INOPMA). The key idea is the realization that the correlation sequence of the outputs of a vibrating structure may be considered as an impulse response but with a certain phase shift [5]. By taking the convolution of one sine wave with the correlation sequence we show that a mode is isolated at a characteristic frequency which depends on the damping ratio. By using a force that is in quadrature of phase with the first one, the damping ratio may be estimated which in turn leads to the estimation of the undamped natural frequency.

We investigate in this paper the performance of INOPMA, first on simulated data (SDOF, MDOF), and it is shown that this method provides estimates of the modal parameters with much less variance than subspace algorithm (the balanced realization (BR) algorithm) [7]. Second, 
the method is validated on experimental data and comparable results with the subspace algorithm are obtained.

\section{The Case of a SDOF System}

Consider a SDOF system with undamped natural frequency $\omega_{n}$ and damping ratio $\zeta$, excited with a random force with spectrum of amplitude $S_{0}$. The correlation sequence of the output $y(t)$ is given by [8]:

$$
R(\tau)=\frac{S_{0} \omega_{n}}{4 \zeta} e^{-\zeta \omega_{n} \tau}\left[\cos \left(\omega_{d} \tau\right)+\frac{\zeta}{\sqrt{1-\zeta^{2}}} \sin \left(\omega_{d} \tau\right)\right]
$$

where $\omega_{d}=\omega_{n} \sqrt{1-\zeta^{2}}$.

This correlation sequence is a decaying sinusoid but with a certain phase shift that depends on the damping ratio. Therefore it may be considered as an impulse response except that the phase shift needs to be taken into account.

Let us consider now the convolution of this correlation sequence with a pure sine wave with driving frequency $\omega$, that is, $f(t)=\sin (\omega t)$, and call the result $x(t)$ which is also a sine wave at the same frequency.

The transfer function $G(s)=x(s) / f(s)$ is given by [8]:

$$
G(s)=\frac{S_{0}}{4 \zeta} \frac{s+2 \varsigma \omega_{n}}{s^{2}+2 \zeta \omega_{n} s+\omega_{n}^{2}} .
$$

The tangent of the phase angle of this transfer function can be shown to be as follows:

$$
\tan (\phi)=-\frac{\omega}{2 \zeta \omega_{n}^{3}}\left[\omega^{2}-\omega_{n}^{2}\left(1-4 \zeta^{2}\right)\right] .
$$

This phase angle is exactly zero at the following frequency:

$$
\omega^{*}=\omega_{n} \sqrt{1-4 \zeta^{2}}
$$

By varying the driving frequency $\omega$ and computing the phase angle between the input and the output, this frequency can be identified exactly. We call this property the phase resonance. This is similar to the phase resonance property used in modal appropriation [6]. The convolution done between the force and the correlation sequence may be thought of as applying a harmonic force to a certain structure.

Notice that, however, since the damping ratio is not known, it is not possible at this stage to identify $\omega_{n}$. It is possible however to identify $\omega^{*}$ exactly as this is the frequency at which the phase becomes zero. Next the damping ratio needs to be estimated. With $\zeta$ known (4) yields an estimate of $\omega_{n}$.

Once $\omega^{*}$ is identified, let us take the convolution of the correlation sequence with a harmonic signal that is in quadrature of phase (at pi/2) with respect to the first one and with amplitude $\alpha$; that is, $g(t)=(1+j \alpha) f(t)$.

We can show that the phase resonance now occurs at the frequency $\widetilde{\omega}^{*}$ given by

$$
\widetilde{\omega}^{*}=\omega^{*} \sqrt{1+\alpha \zeta \beta(\zeta)}
$$

where $\beta(\zeta)$ is given by

$$
\beta(\zeta)=\left(1-4 \zeta^{2}\right)^{-(3 / 2)}
$$

By varying $\alpha$ and computing the new phase resonance frequencies, the damping ratio may be estimated by solving the following equation:

$$
\frac{1}{\omega^{* 2}} \frac{\partial \widetilde{\omega}^{* 2}}{\partial \alpha}=\xi \beta(\zeta)
$$

For exact estimation of $\zeta$ one can fit (7). However, for a wide range of damping ratios, the function $\beta(\zeta)$ is almost 1 . A very good estimate of $\zeta$ may be given by

$$
\zeta \approx \frac{1}{\omega^{* 2}} \frac{\partial \tilde{\omega}^{* 2}}{\partial \alpha}
$$

Once the damping ratio is estimated, the natural frequency may be estimated from (4).

\section{Simulation Study}

In this section we consider a simulation study of the presented method. In particular we consider a single-input singleoutput system (SISO) and a multi-input single-output system (MISO). We show that the INOPMA method outperforms subspace methods (the BR algorithm) by providing modal parameters with much less bias and variance.

3.1. SISO Systems. We consider here a SDOF system excited with white noise. The mass, damping, and stiffness values used are, respectively, $m=100 \mathrm{~kg}, c=80 \mathrm{Ns} / \mathrm{m}$, and $k=$ $100 \times 10^{3} \mathrm{~N} / \mathrm{m} .2000$ data points were simulated and $2 \%$ noise was added to the output. A total of 60 experiments were performed where at each experiment a different realization of the excitation as well as the noise is used. The subspace algorithm was used for the extraction of the modal parameters with truncation of the Hankel matrix at the exact model order. Initially, at the first run, the empirical variances of the modal parameter estimates are set to zero. Then at each successive run the set of identified modal parameters increases and is used to compute the empirical variance of that set. As the runs increase the number of modal parameters increases and better estimates of the variances (variance of the set of the estimated modal parameters) are obtained which will eventually converge. The results are shown in Figures 1 and 2. It is obvious from the figures that INOPMA presents much less variance than the subspace algorithm. In order to study the bias on the modal parameters, the relative error (the exact value minus the estimated value over the exact value) on the frequency and damping is computed for 60 simulation runs. Inspection of Figures 3 and 4 shows that INOPMA outperforms the subspace algorithm.

3.2. MISO Systems. We consider here a simulation study of a MISO system. In particular, a 2DOF system is considered 


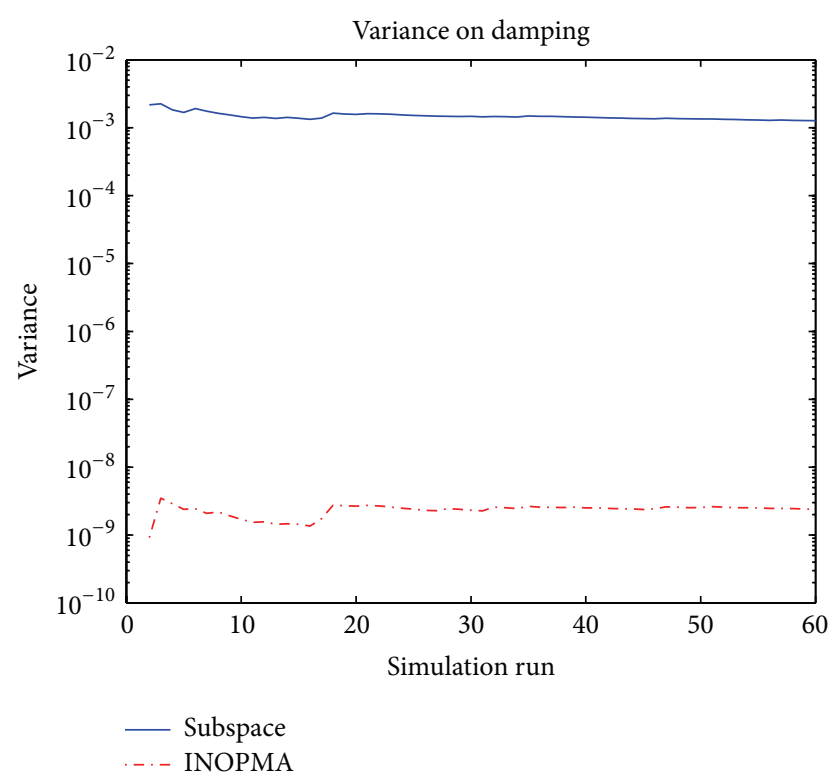

FIgURE 1: Variance on damping: SDOF case.

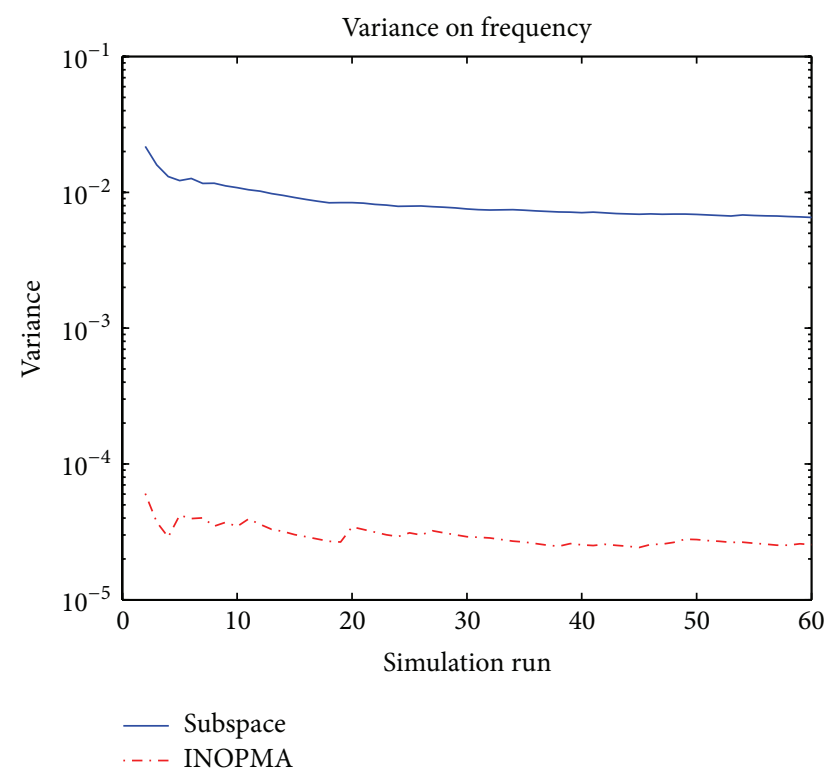

FIgURE 2: Variance on frequency: SDOF case.

but only 1 output is chosen in the identification process. The mass, damping, and stiffness matrices used are, respectively,

$$
\begin{aligned}
& M=\left[\begin{array}{cc}
100 & 0 \\
0 & 100
\end{array}\right], \quad C=\left[\begin{array}{cc}
200 & -100 \\
-100 & 300
\end{array}\right], \\
& K=\left[\begin{array}{cc}
200 & -100 \\
-100 & 300
\end{array}\right] \times 10^{3} .
\end{aligned}
$$

The same experiments as for the SDOF system are performed. The results are shown in Figures 5 and 6 where it is obvious that the INOPMA method again presents much less variance than the subspace algorithm. The bias on the first mode is illustrated in Figures 7 and 8.

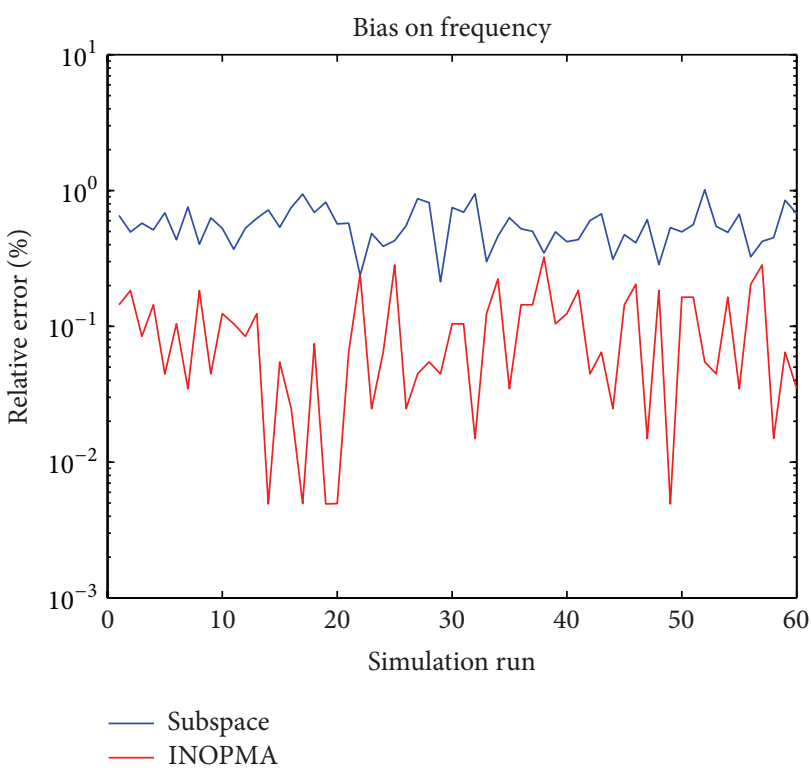

FIgURE 3: Absolute value of the relative error on the frequency.

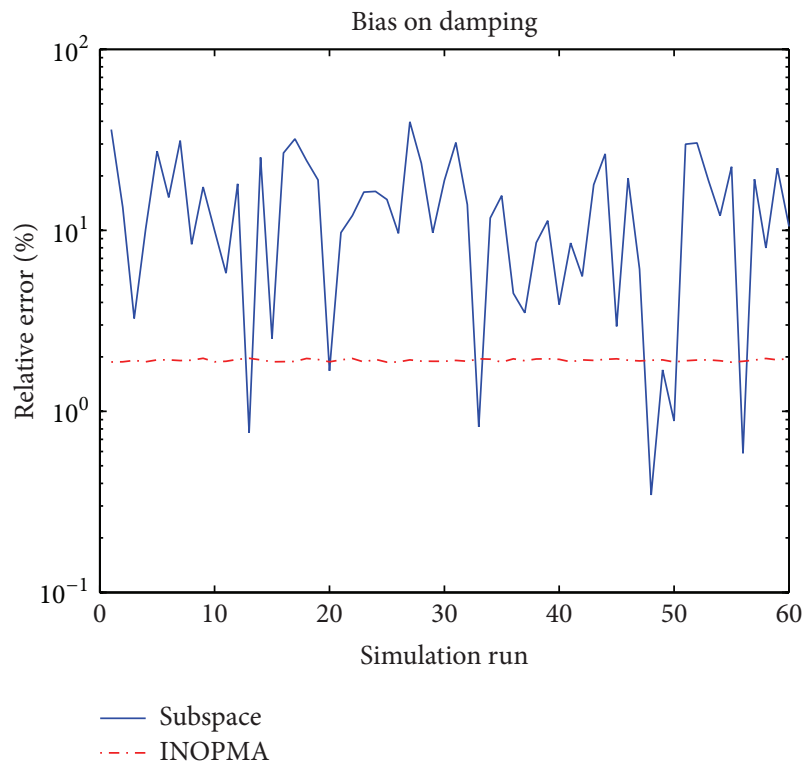

FIgURE 4: Absolute value of the relative error on damping.

\section{Experimental Study}

The structure considered in this study consists of a suspended aircraft skeleton structure used extensively in modal identification studies [9] (Figure 9). The structure was excited at two different locations using random noise inputs, and 7 accelerometers were used to measure the time responses. The analysis was performed in the $0-128 \mathrm{~Hz}$ frequency range and 5000 data points per channel were collected at $256 \mathrm{~Hz}$ sampling frequency. All 7 sensors were used to identify the experimental natural frequencies, damping ratios, and mode shapes using the 5000 data points. The subspace algorithm 


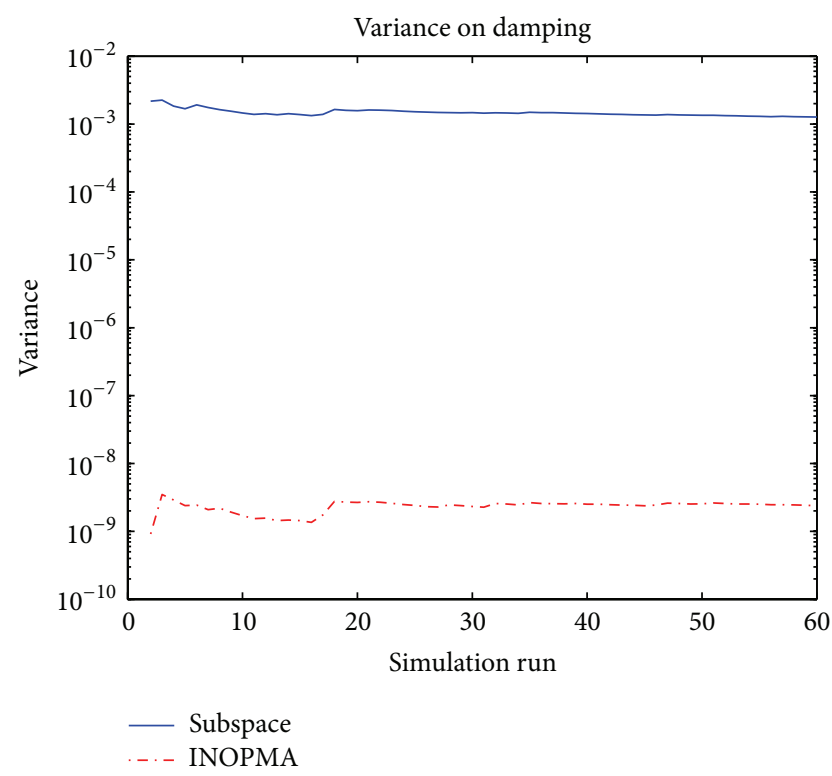

FIGURE 5: Variance on damping: MISO system.

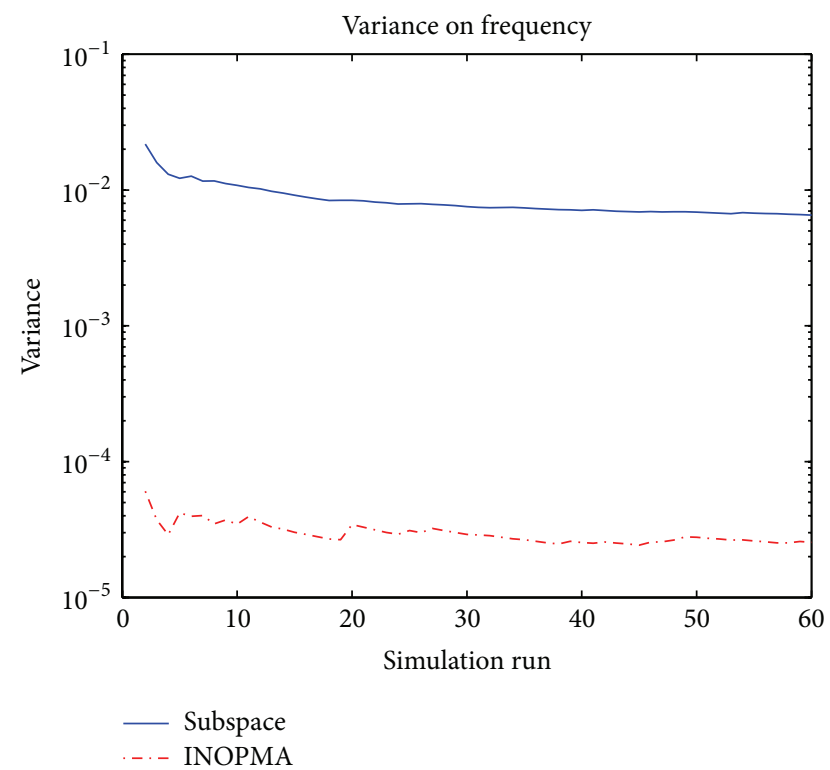

FIGURE 6: Variance on frequency: MISO system.

(BR) was used for the identification. The results are given in Table 1 [9].

For INOPMA, all 5000 data points were used to compute the data correlations at 128 lags. Only one sensor at a time is used for identification. Sensors 3 and 5 were used and give similar results. The results are reported in Table 1 and it can be seen that they compare well with those of the subspace algorithm.

\section{Conclusion}

A new numerical modal appropriation based method for use with in-operation modal analysis (INOPMA) has been

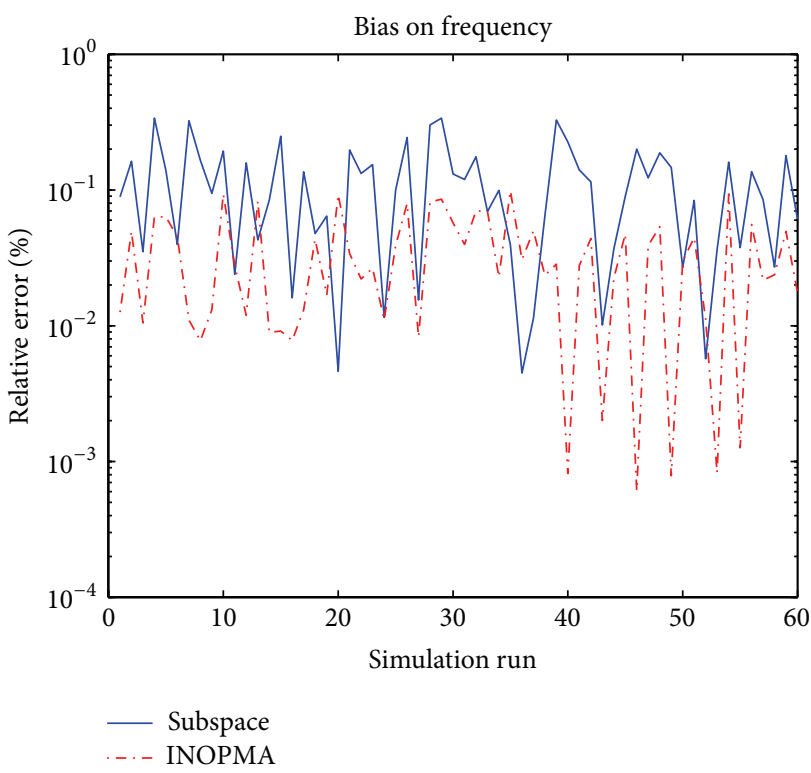

FIgURE 7: Absolute value of the relative error on the frequency.

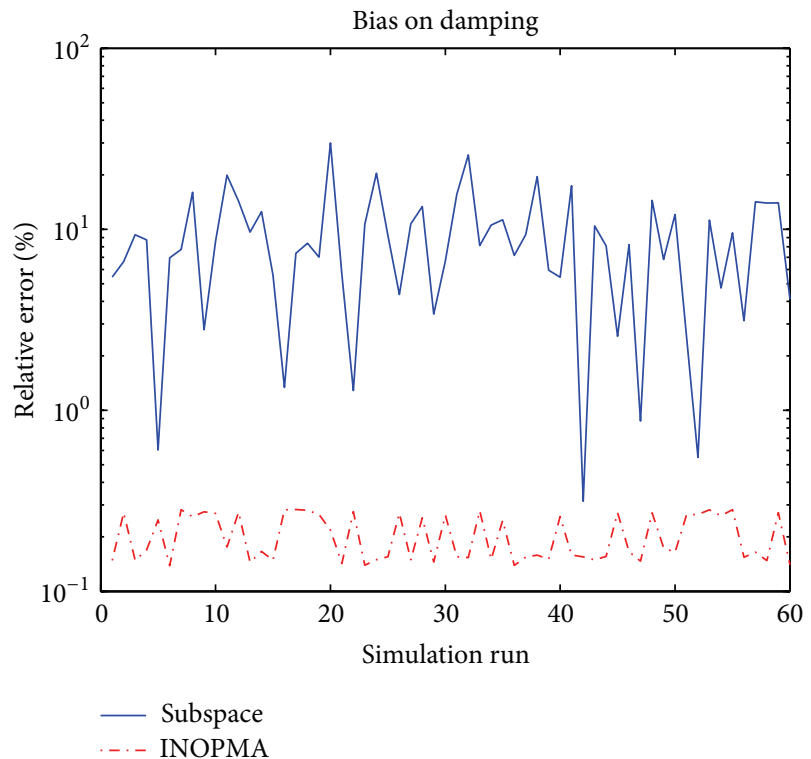

Figure 8: Absolute value of the relative error on damping.

derived. The key concept is the realization that the output correlation sequence is a decaying sinusoid with a certain phase shift and therefore may be considered as an impulse response. The convolution of this correlation sequence with a pure sine wave allows the isolation of the mode at a characteristic frequency which depends on the damping ratio. By using a force in quadrature of phase with a sine it is possible to estimate the damping ratio which in turn allows the estimation of the undamped natural frequency. This approach is validated on simulated data and it is shown that the modal parameters determined in this way present much less variance and bias than subspace based identified methods. The proposed method is also validated 

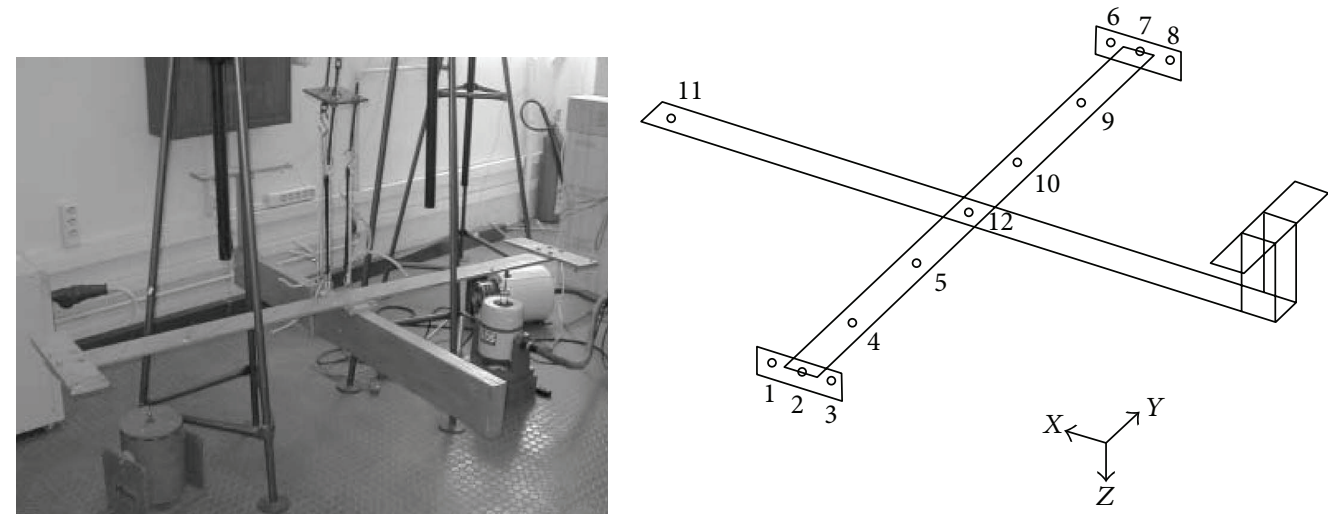

FIGURE 9: Aircraft skeleton structure [9].

TABLE 1: Identification results: INOPMA versus subspace.

\begin{tabular}{lcccc}
\hline \multirow{2}{*}{ Mode } & \multicolumn{2}{c}{ Frequency $(\mathrm{Hz})$} & \multicolumn{2}{c}{ Damping $(\%)$} \\
& INOPMA & Subspace & INOPMA & Subspace \\
\hline 1 & 3.93 & 3.71 & 4.95 & 4.9 \\
2 & 6.15 & 6.13 & 3.92 & 3.6 \\
3 & 16.09 & 16.06 & 4.33 & 4.05 \\
4 & 21.88 & 21.71 & 5.01 & 5.73 \\
5 & 39.86 & 39.87 & 0.37 & 0.38 \\
6 & 43.96 & 44.33 & 0.04 & 0.03 \\
7 & 54.36 & 54.35 & 0.16 & 0.11 \\
8 & 55.42 & 55.42 & 0.29 & 0.22 \\
9 & 58.36 & 58.44 & 0.4 & 0.38 \\
10 & 68.67 & 69.41 & 1.5 & 1.12 \\
11 & 80.24 & 80.06 & 0.11 & 0.09 \\
12 & 99.93 & 100.25 & 0.12 & 0.11 \\
\hline
\end{tabular}

on experimental data and it is shown that the results compare well with the subspace method. One of the advantages of this method is the fact that there is no need of a stabilization diagram as required in many modal identification methods (subspace). The extension of this approach to MIMO systems is on-going.

\section{Conflict of Interests}

The authors declare that there is no conflict of interests regarding the publication of this paper.

\section{References}

[1] M. J. Desforges, J. E. Cooper, and J. R. Wright, "Spectral and modal parameter estimation from output-only measurements," Mechanical Systems and Signal Processing, vol. 9, no. 2, pp. 169186, 1995.

[2] E. Luz and J. Wallaschek, "Experimental modal analysis using ambient vibration," The International Journal of Analytical and Experimental Modal Analysis, vol. 7, no. 1, pp. 29-39, 1992.

[3] B. Peeters and G. de Roeck, "Reference-based stochastic subspace identification for output-only modal analysis," Mechanical Systems and Signal Processing, vol. 13, no. 6, pp. 855-878, 1999.
[4] F. Shen, M. Zheng, D. Feng Shi, and F. Xu, "Using the crosscorrelation technique to extract modal parameters on responseonly data," Journal of Sound and Vibration, vol. 259, no. 5, pp. 1163-1179, 2003.

[5] G. H. James, T. G. Carne, and J. P. Lauffer, "The natural excitation technique (NExT) for modal parameter extraction from operating structures," International Journal of Analytical and Experimental Modal Analysis, vol. 10, no. 4, pp. 260-277, 1995.

[6] E. Balmes, C. Chapelier, P. Lubrina, and P. Fargette, "An evaluation of modal testing results based on the force appropriation method," in Proceedings of the International Modal Analysis Conference, Orlando, Fla, USA, 1996.

[7] L. Hermans and H. D. van Auweraer, "Modal testing and analysis of structures under operational conditions: industrial applications," Mechanical Systems and Signal Processing, vol. 13, no. 2, pp. 193-216, 1999.

[8] L. Meirovitch, Elements of Vibration Analysis, McGraw-Hill, 1986.

[9] V. Papakos and S. D. Fassois, "Multichannel identification of aircraft skeleton structures under unobservable excitation: a vector AR/ARMA framework," Mechanical Systems and Signal Processing, vol. 17, no. 6, pp. 1271-1290, 2003. 

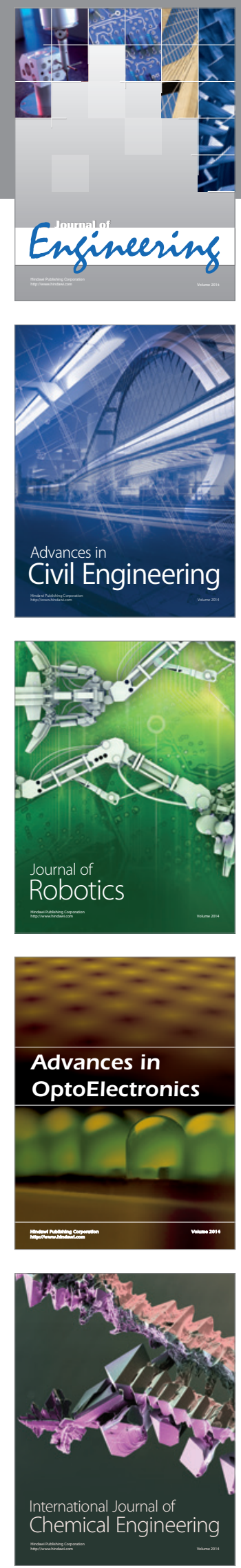

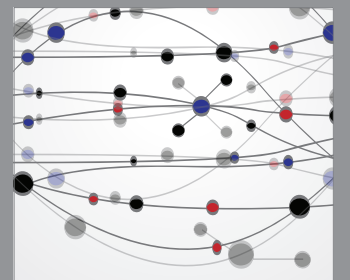

The Scientific World Journal
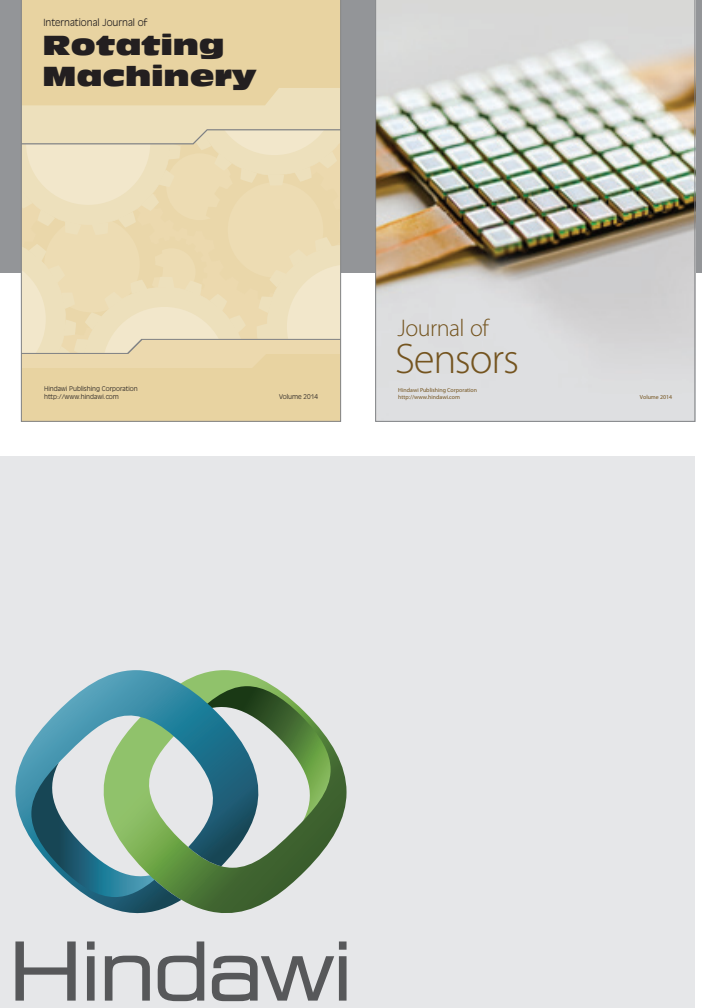

Submit your manuscripts at http://www.hindawi.com
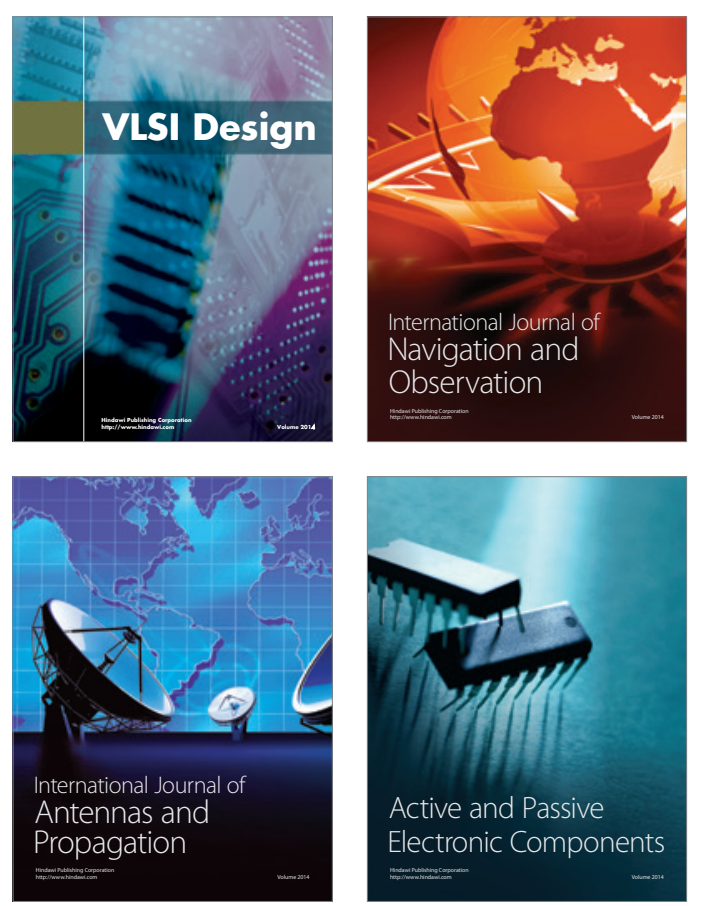
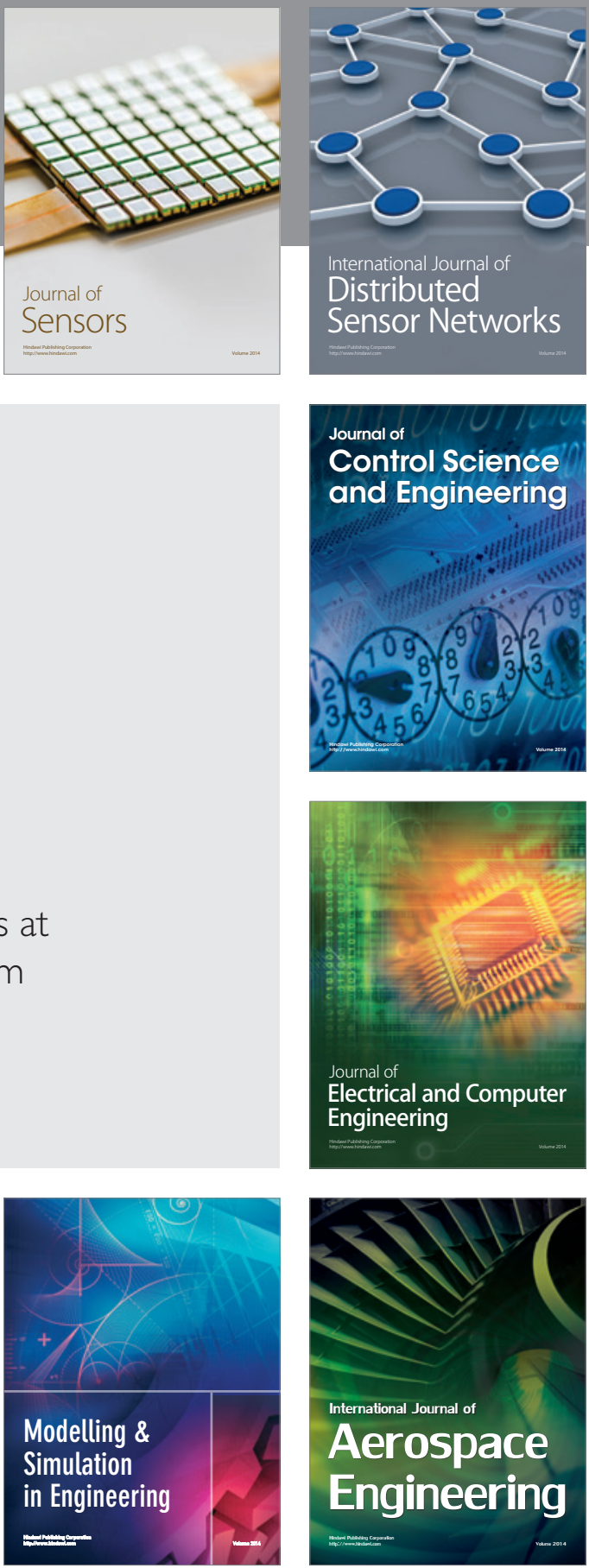

Journal of

Control Science

and Engineering
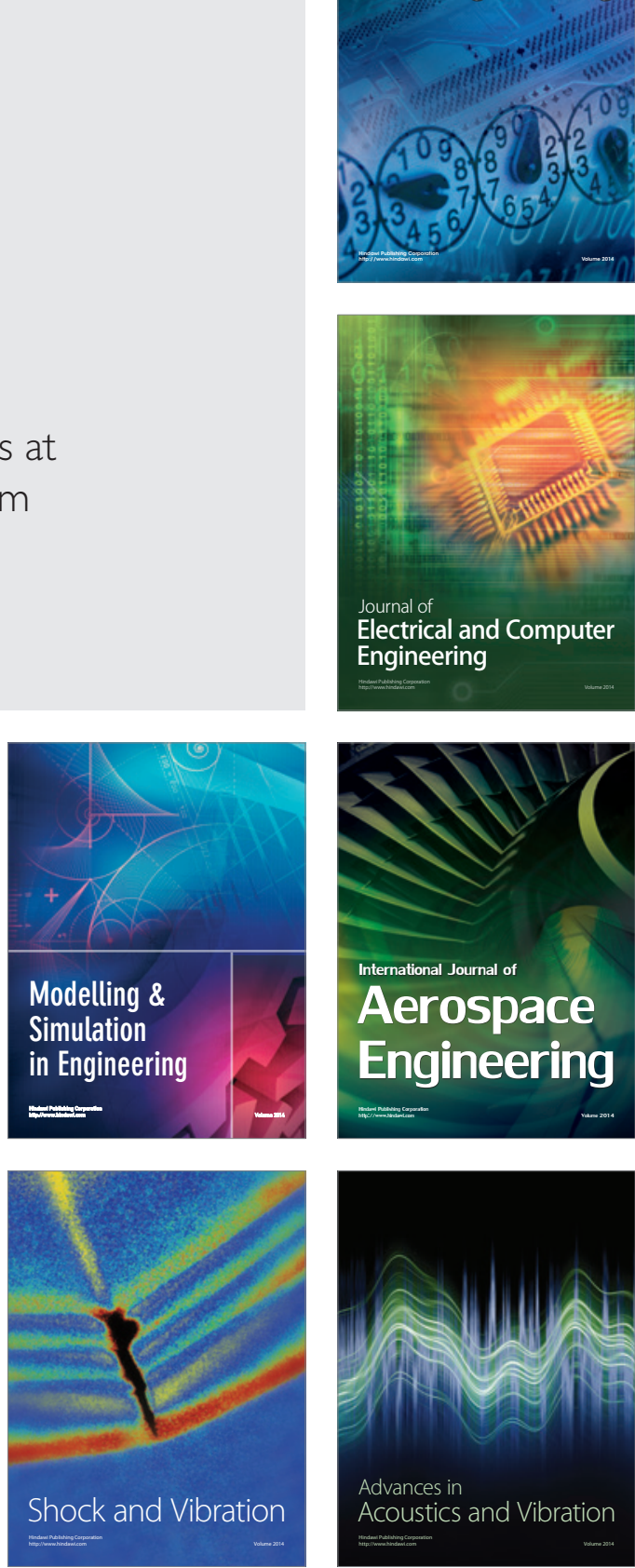